\title{
Concepción de un simulador para el entrenamiento de operadores de centrales hidroeléctricas y desarrollo del arranque de un generador
}

\section{Conception of a simulator for training operators of hydropower plants and development of the starting of a generator}

\author{
Juan Carlos García-Arredondo ${ }^{1,2}$, José Ramírez-Scarpetta $^{3}$ \\ ${ }^{1}$ Universidad del Valle, Colombia. Email: juangarcia1439@gmail.com \\ ${ }^{2}$ Institución Universitaria Antonio José Camacho, Colombia. Email: jcgarcia@admon.uniajc.edu.co \\ ${ }^{3}$ Universidad del Valle, Colombia. Email: jose.ramirez@correounivalle.edu.co
}

RECIBIDO: Febrero 12, 2017. ACEPTADO: Marzo 30, 2017. Versión FINAL: Mayo 24, 2017.

\begin{abstract}
RESUMEN
En el entrenamiento de operadores de centrales hidroeléctricas, el operador aprendiz puede realizar una mala maniobra, con salidas de operación o daños en los equipos, con los costos que ello implica para la empresa. Un simulador permite el entrenamiento de aprendices en condiciones de seguridad en la operación y además permite entrenar a los operadores en situaciones inusuales con condiciones críticas de operación o de falla. Este artículo presenta la concepción de un simulador de entrenamiento para operadores de centrales hidroeléctricas. Inicialmente, se definen los sistemas y equipos y las condiciones para la operación del generador. Después, se presentan los requerimientos para el simulador y los casos de uso; con ellos, se diseña la arquitectura software y hardware y las interfaces con el usuario. Finalmente, se muestra el desarrollo y prueba de las interfaces para el arranque del generador, mediante el uso del software de programación LabVIEW®. En las pruebas se aprecia a las interfaces que se conectan con los modelos matemáticos de los subsistemas. Esta conexión de los modelos con las interfaces del simulador, puede ser vista por el cambio en el estado de las variables que conforman las interfaces.
\end{abstract}

PALABRAS CLAVE: Simulador de entrenamiento, Centrales hidroeléctricas, Modelos, Grupo de generación, LabVIEW®, Requerimientos, Interfaz, Casos de uso, Operador.

\begin{abstract}
In the training of operators of hydropower plants, the apprentice operator could make a bad handling, which could stop the whole operation of the plant or damage the equipment, generating unwanted costs to the enterprise. A simulator allows training of apprentices in safe conditions during the operation and allows the training of operators in unusual situations with critical operating conditions or failure. This paper presents the design of a training simulator for operators of hydropower plants. Initially, systems and equipment are defined as well as the initial conditions for the correct operation of the generator. Then, the requirements for the simulator and use cases are presented and the software and hardware architecture and user interfaces are designed. Finally, the development and testing of interfaces for the starting of the generator are shown by using LabVIEW@ programming software. Tests show the interfaces that connect with mathematical models of subsystems. This connection of models with the interfaces of the simulator can be seen due to the change in the state of the variables that are part of the interfaces.
\end{abstract}

KEYWORDS: Training simulator, Hydropower, Models, Generation group, LabVIEW, Requirements, Interface, Use Case, Operator.

Este artículo puede compartirse bajo la licencia CC BY-ND 4.0 y se referencia usando el siguiente formato: J. C. García-Arredondo, J. M. RamírezScarpetta, "Concepción de un simulador para el entrenamiento de operadores de centrales hidroeléctricas y desarrollo del arranque de un generador", UIS Ingenierías, vol. 16, no. 2, pp. 105-118, Julio-Diciembre 2017. Doi: https://doi.org/10.18273/revuin.v16n2-2017010 


\section{INTRODUCCIÓN}

Una mala maniobra o una respuesta lenta ante una falla en el sistema de generación de energía eléctrica, perjudica a los componentes eléctricos y mecánicos del control de generación, con problemas para las empresas de energía, ya que implica no brindar a los usuarios la confiabilidad que se espera del sistema. Una solución para ayudar a evitar estas deficiencias, es el entrenamiento de operadores de la sala de control. No es técnica ni económicamente viable entrenar al operador con el proceso físico, por las limitaciones operativas y la calidad del servicio y por los costos que implica tener condiciones operativas especiales. Por lo tanto, es importante contar con simuladores para el entrenamiento y la capacitación del personal de operación de las centrales.

El uso de simuladores para el entrenamiento y la capacitación del personal de operación de las centrales. El uso de simuladores para el entrenamiento de operadores que maniobran un sistema, ha mostrado ser una de las mejores formas de capacitar al personal [1] y desarrollar procedimientos operacionales. Además, se ha demostrado su utilidad y una relación costo/beneficio muy importante, ya que contribuyen fuerte $y$ efectivamente a lograr ahorros considerables a las empresas eléctricas en el contexto de "reducción de pérdidas técnicas". Los ahorros se obtienen por el buen desempeño de operadores entrenados en apoyar a aumentar la disponibilidad de los grupos de generación, en alargar la vida útil de sus equipos, en aumentar la eficiencia energética y en apoyar en la reducción del impacto ambiental. Por otro lado, un estudio de EPRI establece que en promedio, por contribuir en los rubros mencionados, se logran ahorros de hasta 3000 USD/MW instalado/año. Por estas razones, empresas internacionales proveedoras de simuladores establecen que si el entrenamiento de operadores se lleva a cabo sólo a nivel teórico, se logra cuando mucho un aprovechamiento de $25 \%$ al $30 \%$, si se utilizan simuladores genéricos un $50 \%$ a $55 \%$, y si se utilizan simuladores réplica, hasta $80 \%$ a $100 \%$ [2]. Por todo lo anterior, Varios simuladores han sido desarrollados, por ejemplo en el área eléctrica en [1] se desarrolló un simulador de entrenamiento para el control de tensión y estabilidad del sistema de transmisión de $735 \mathrm{kV}$, reproduce la conducta del proceso, pero no reproduce la sala de control MFDS (Model Forward for Design Simulators).

En la implementación del simulador se obtiene ecuaciones de equilibrio más no son ecuaciones que representen el comportamiento dinámico del sistema. En [3] se realiza un simulador en tiempo real para certificar y entrenar operadores de centrales y subestaciones eléctricas. Para la modelación y simulación, se utilizó el Toolbox de MATLAB Simpower Systems que cuenta con los modelos para los diferentes subsistemas. La interface gráfica no es del tipo SCADA que permita enfrentar al operador con pantallas reales del proceso. En [4] se realiza un simulador de entrenamiento para operadores de centrales de energía geotérmica. Los modelos matemáticos están escritos en lenguaje Fortran y el modelo de sistema de control distribuido (DCS) fue desarrollado utilizando un modelamiento gráfico escrito en lenguaje C. En [5] se desarrolló para XM, un simulador de entrenamiento tipo MFDS que reproduce el comportamiento del sistema interconectado nacional colombiano. El simulador utiliza algoritmos matemáticos para el modelado de los subsistemas y utiliza una arquitectura del tipo SCADA. En [6] se desarrolló un FSS (Full Scale Simulators) o simulador de alcance total para el entrenamiento en la operación de centrales termoeléctricas de $800 \mathrm{MW}$ de la Industria Eléctrica de Rusia. El simulador utiliza algoritmos matemáticos para el modelado de los subsistemas y el uso de un sistema de control distribuido (DCS); para centrales hidráulicas, objeto de este trabajo, en [7] se presenta el desarrollo de un simulador para el entrenamiento tipo FSS para la enseñanza e investigación en la operación de una central hidroeléctrica. El simulador es realizado para una central específica. Para el modelado se tiene en cuenta el software de FORTRAN 77, que no es amigable para el uso de interfaces; en [8] se desarrolló un simulador tipo MFDS que sirve como entrenador para operarios de la central. El modelado de los subsistemas de la central, son obtenidos con base en leyes físicas y el sistema de control es del tipo DCS.; en [9], se presenta un sistema de entrenamiento FSS estático para una pequeña central hidroeléctrica. Por ser un sistema estático no tiene en cuenta la influencia de modelos matemáticos de los diferentes subsistemas, pero utiliza una arquitectura del tipo SCADA.

Respondiendo a estas necesidades de apoyo al trabajo en las centrales hidroeléctricas, en este artículo se concibe un simulador de entrenamiento, que brinde apoyo a la formación de operadores y desarrollo del arranque de un grupo de generación.

\section{PROCESO DE GENERACIÓN DE ENERGÍA ELÉCTRICA EN UNA CENTRAL HIDROELÉCTRICA}

En esta sección, se hace una breve descripción del proceso de generación de energía, en una central hidroeléctrica de gran potencia. En una central de se tiene almacenada una cantidad de agua en un embalse con 
energía potencial que se convierte en cinética al circular desde cierta altura por una tubería de conducción que al final se estrecha para aumentar la presión del agua y de esta forma pasar por la válvula de entrada hasta la turbina para ponerla en marcha y así convertir la energía hidráulica en energía mecánica. Esta energía provoca un movimiento de rotación del generador que transforma dicha energía en electricidad. El conjunto formado por estas dos máquinas se conoce como grupo hidroeléctrico. Como la generación de energía se realiza continuamente, el proceso es clasificado como continuo. En la Figura 1, se ilustran los principales componentes.

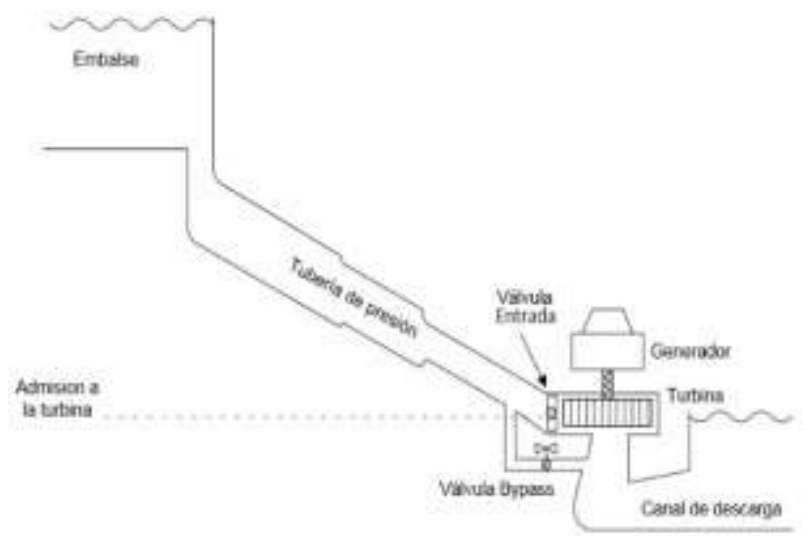

Figura 1. Esquemático para el proceso de generación de energía eléctrica. Fuente. [10].

\subsection{Sistemas y equipos del proceso}

En este ítem, se describen los principales sistemas y equipos que cumplen una función específica, para el funcionamiento y la puesta en servicio del grupo hidroeléctrico. Ver la Figura 2. A continuación en las subsecciones, se realiza esta descripción.

\subsubsection{Sistema hidráulico.}

Es el conjunto de elementos que generan fuerza hidráulica: volumen de aceite presurizado por aire comprimido en el tanque acumulador, tanque de depósito de aceite, sistema de electroválvulas, bombas eléctricas, instrumentación de control, tuberías de carga y descarga del sistema, etc.; acciona los álabes del distribuidor, la válvula de entrada y la válvula de desvío.

\subsubsection{Sistema neumático.}

Es el conjunto de elementos que generan fuerza neumática: tanque de aire comprimido, electroválvulas, compresores, instrumentación de control, tuberías, etc.; principalmente soporta el sistema hidráulico del gobernador, también otros subsistemas como la protección contra incendios.

\subsubsection{Sistema de puesta a tierra.}

Es una ligazón metálica sin fusibles ni protección, entre elementos de la central (penstok, equipos, tuberías de agua, etc.) y electrodos enterrados, para que no existan voltajes peligrosos y se permita el paso a tierra de corrientes de falla.

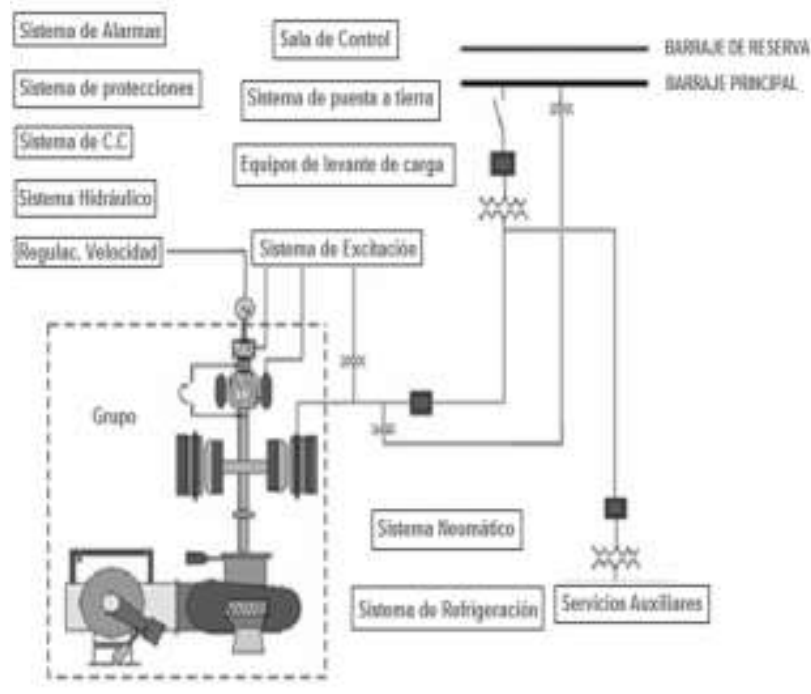

Figura 2. Sistemas del proceso de generación. Fuente. [11] .

\subsubsection{Sistema de servicios auxiliares de corriente alterna.}

Este sistema alimentado de una fuente externa o de los mismos grupos, provee la fuente de corriente alterna para el suministro de energía a los motores y los transformadores de distribución para el alumbrado de la casa de máquinas.

\subsubsection{Sistema de servicios auxiliares en corriente continua.}

Se compone de cargadores de baterías, banco de baterías, barraje colector de fuentes, interruptor general del sistema, barraje de distribución, interruptores de circuitos y las cargas propiamente dichas. Con este sistema se tiene la seguridad del suministro para la operación de los controles, relés de protección, anuncio y señalización y la iluminación de emergencia.

\subsubsection{Sistema de levante de carga.}

Puente grúa para el montaje y desmontaje de los equipos.

\subsubsection{Sala de control.}

Es el lugar en la casa de máquinas donde se centraliza la operación, control, protección, medición, comunicación, maniobra, y todo acto que afecte el desempeño de todos los equipos, sistemas y componentes que se encuentran en servicio o reserva.

\subsubsection{Sistema de alarmas.}


Recoge las novedades que se presentan en los sistemas durante el servicio; se señalizan en los tableros de sala de control y en los tableros de los equipos, acompañados de sonido de sirena; el sistema consta de paneles porta pilotos, relés auxiliares, sirena e interruptores de comprobación.

\subsubsection{Sistema de protecciones.}

Señalizado en la sala de control; saca de servicio equipos o componentes, en caso de avería grave. Incluye los sistemas de seguridad contra incendios.

\subsubsection{Sistema grupo.}

La unión de la turbina y el generador forma un grupo. Un generador eléctrico convierte energía mecánica en energía eléctrica. Al generador, se hace girar el rotor por medio de una fuerza externa, generalmente proporcionada por una turbina impulsada por un flujo ya sea de agua, vapor o por la fuerza producida por un motor de combustión interna.

\subsubsection{Sistema regulador de velocidad.}

Regula la velocidad en el eje de la turbina y en consecuencia la frecuencia del sistema; como la frecuencia es dependiente del balance de potencia en el sistema, los gobernadores de velocidad también controlan la potencia del grupo. La frecuencia y la potencia eléctrica cambian constantemente en un sistema de potencia debido a las variaciones en la carga, disparo de plantas generadoras o fallas inherentes en el sistema. También vela por la seguridad de la turbina y tubería de presión y participa en las funciones de mando como arranque, sincronización y parada.

\subsubsection{Sistema de excitación.}

Proporciona la corriente continua al devanado de campo del generador sincrónico, para mantener el voltaje en terminales constante, controla el flujo de potencia reactiva; complementariamente, aumenta la estabilidad del sistema de potencia y proporciona funciones de protección y limitación al generador y al excitador.

A continuación, se hace una descripción de los principales modelos del proceso de generación de energía.

\section{MODELOS MATEMÁTICOS DE LOS PRINCIPALES SISTEMAS DEL PROCESO}

Para que el simulador de entrenamiento diseñado en este trabajo, tenga un comportamiento en tiempo real similar al proceso de generación de energía, se consideran los modelos matemáticos del control de excitación y velocidad. Adicionalmente, el del generador.

\subsection{Modelo para el control de excitación y velocidad}

En este caso, se utilizan los modelos del control de excitación y el control de velocidad, obtenidos en [12] [13]. Estos modelos han mostrado buenos resultados para diferentes condiciones de operación al ser validados en estos trabajos, para estudios dinámicos de una central de la región.

\subsection{Modelo del generador sincrónico}

Para el generador, se utiliza el modelo propuesto en el toolbox de sistemas de potencia del software simulink de Matlab [14]. El modelo se denomina Synchronous Machine pu Standard. Para el régimen en vacío, es indispensable que a la salida del modelo se coloque una pequeña carga ( $10 \%$ de la potencia activa nominal). Esto, porque el modelo está implementado como una fuente de corriente.

\section{OPERACIÓN DEL GRUPO}

En esta parte, inicialmente se hace una descripción de las condiciones que deben cumplirse para la operación de un Grupo Generador. Se continúa con la secuencia de pasos que debe seguir el operador de una Central Hidroeléctrica, para realizar el proceso de arranque, sincronización y la parada. Finalmente, se hace un análisis funcional $\mathrm{y}$ tecnológico del sistema de generación, con base en el enfoque de los proyectos de automatización.

Para la operación de las centrales hidroeléctricas se exige un riguroso conocimiento de las maniobras involucradas. Las maniobras que todo operario debe conocer a cabalidad son: el arranque, la sincronización y la parada, según [15].

Para realizar el arranque de un grupo turbina-generador debe seguirse una secuencia lógica que permita que éste sea adecuado. Después de efectuado el arranque se procede a la sincronización con el sistema de potencia. Las maniobras de arranque y sincronización se hacen en conjunto. Finalmente, la parada de un grupo puede producirse por: fallas internas del grupo, por operación o mantenimiento. En este trabajo se describe el arranque, para su posterior implementación.

\subsection{Secuencia de arranque del grupo}

En la Figura 3 se muestra un diagrama lógico, con las condiciones preliminares que deben verificarse inicialmente con sus correspondientes estados y valores para los equipos, antes de realizar el arranque. Para la comprensión de los diagramas lógicos, en la Figura 4 se muestran las convenciones que se usan. Después, en la Figura 5 se muestra en un diagrama de bloques el procedimiento a seguirse para el arranque de un grupo. 
Con base en estos diagramas, más adelante, se desarrolla la interface para el arranque del grupo.

\subsection{Sincronización}

Después de efectuado el arranque del grupo, se procede a la sincronización de éste con el sistema de potencia. En este trabajo no se hace la sincronización.

\subsubsection{Condiciones para la sincronización.}

Las condiciones para conectar el grupo al Sistema Interconectado Nacional (SIN) son las siguientes:
- El voltaje del grupo a conectar, debe ser igual al voltaje de la red.

- Las frecuencias de los sistemas a acoplar no deben ser mayor del $1 \%$.

- La secuencia de las fases debe ser igual.

- El ángulo de fase del grupo a conectar debe ser igual al de la red.

Las maniobras de arranque y sincronización se hacen en conjunto.

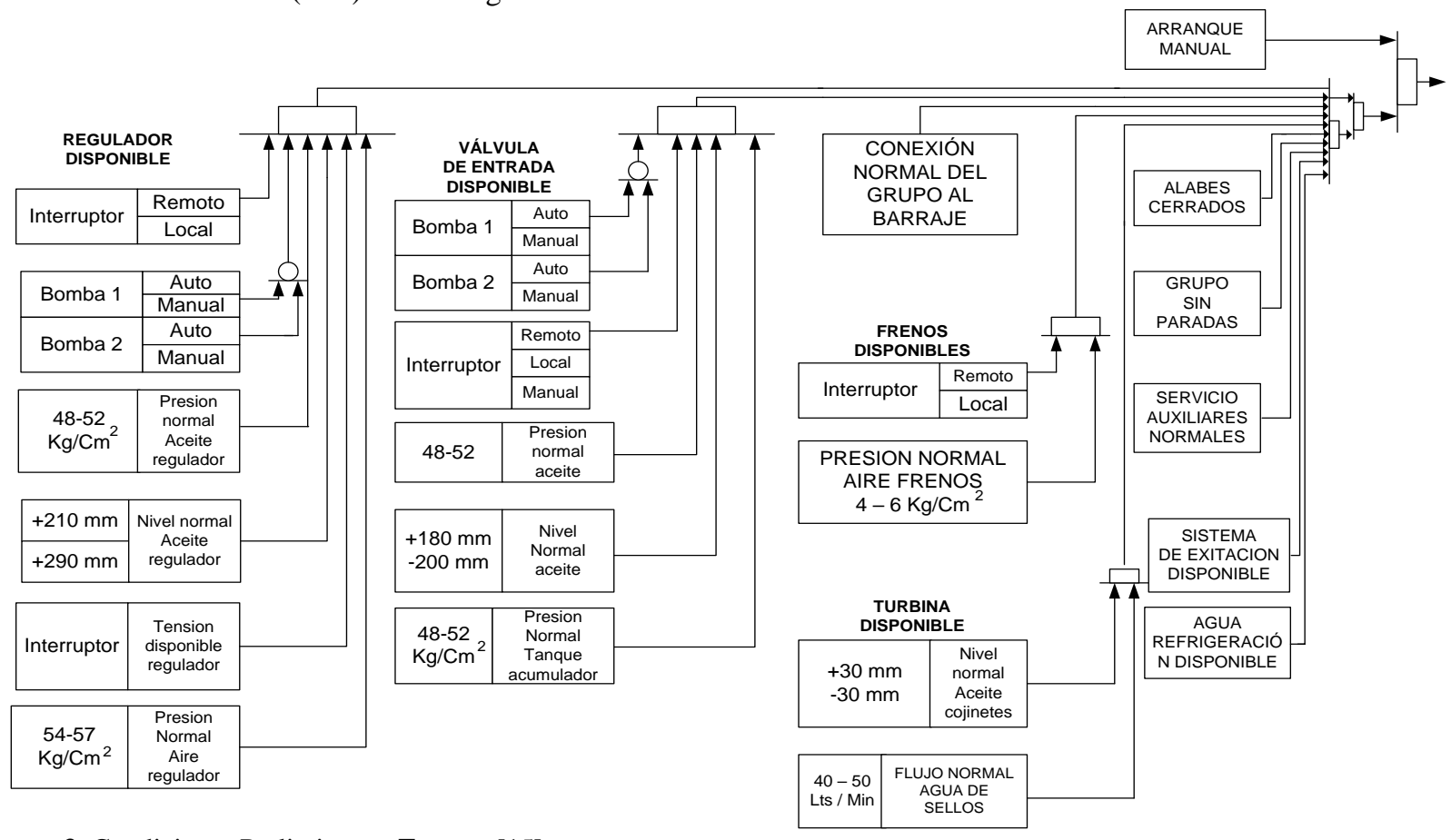

Figura 3. Condiciones Preliminares. Fuente. [15].

\subsection{Secuencia de parada del grupo}

El control en una central, permite que una orden de parada se ejecute estando el grupo con carga activa o reactiva. Inmediatamente la carga baja a cero y el interruptor principal se abre. Para realizar la parada, el mando de arranque debe estar en el modo manual o automático, la secuencia que se desencadena es la contraria a la de arranque. En este trabajo no se hace la parada del grupo. Se realiza la descripción, para que sirva de base para los desarrollos futuros del simulador.

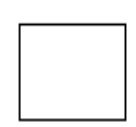

Blaque funional

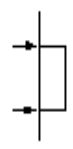

AND

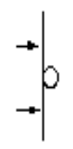

OR

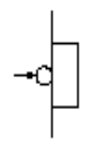

NAND

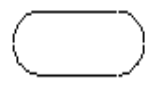

Indicación
Figura 4. Convenciones para los diagramas lógicos.
Una vez se da la orden de parada, el operador de la central hidroeléctrica debe verificar que se haga la siguiente secuencia:

- Abrir del interruptor principal.

- Abrir del interruptor del campo.

- Cerrar los alabes.

- Activar la bomba del cojinete de empuje.

- Cerrar la válvula de entrada.

- Liberar los frenos.

- Parar la bomba de cojinete de empuje.

- Parar la bomba de refrigeración.

- Parada realizada. 
Tabla 1. Arranque y Sincronización del generador.

\begin{tabular}{|c|c|c|c|}
\hline PROCESO & ETAPAS & OPERACIONES & ACCIONES \\
\hline \multirow{18}{*}{$\begin{array}{l}\text { ARRANQUE Y } \\
\text { SINCRONIZACIÓN }\end{array}$} & \multirow{9}{*}{$\begin{array}{l}\text { Revisión } \\
\text { de los } \\
\text { equipos } \\
\text { del } \\
\text { proceso } \\
\text { de } \\
\text { arranque }\end{array}$} & \multirow{3}{*}{$\begin{array}{l}\text { Activar el sistema } \\
\text { hidráulico }\end{array}$} & $\begin{array}{l}\text { Abrir válvula principal de entrada y salida del } \\
\text { generador }\end{array}$ \\
\hline & & & Abrir válvula de agua de sellos y cojinetes \\
\hline & & & $\begin{array}{l}\text { Arranque de bomba de agua de refrigeración del } \\
\text { generador }\end{array}$ \\
\hline & & \multirow{3}{*}{$\begin{array}{l}\text { Activar el sistema } \\
\text { de refrigeración }\end{array}$} & $\begin{array}{l}\text { Funcionamiento de las bombas de agua de } \\
\text { refrigeración de los generadores }\end{array}$ \\
\hline & & & $\begin{array}{l}\text { Funcionamiento de la bomba impulsadora de agua } \\
\text { de refrigeración de los sistemas de la central }\end{array}$ \\
\hline & & & Funcionamiento de los filtros de agua \\
\hline & & \multirow{3}{*}{$\begin{array}{l}\text { Apertura de } \\
\text { válvula de entrada }\end{array}$} & Apertura del By-pass \\
\hline & & & Sacar el sello de servicio \\
\hline & & & Apertura de la válvula principal \\
\hline & \multirow{6}{*}{$\begin{array}{l}\text { Orden de } \\
\text { arranque }\end{array}$} & \multirow{3}{*}{$\begin{array}{l}\text { Activar el sistema } \\
\text { hidráulico }\end{array}$} & Arranque de bomba del cojinete de empuje \\
\hline & & & $\begin{array}{l}\text { Accionamiento de sensores de nivel del regulador } \\
\text { de velocidad }\end{array}$ \\
\hline & & & $\begin{array}{l}\text { Arranque de la bomba de aceite del regulador de } \\
\text { velocidad para el movimiento de los alabes }\end{array}$ \\
\hline & & \multirow{3}{*}{$\begin{array}{c}\text { Funcionamiento } \\
\text { del sistema } \\
\text { neumático }\end{array}$} & $\begin{array}{l}\text { Accionamiento de los sensores de presión del } \\
\text { regulador de velocidad }\end{array}$ \\
\hline & & & $\begin{array}{l}\text { Arranque de los compresores de aire de frenos y } \\
\text { reguladores de velocidad }\end{array}$ \\
\hline & & & Retirar los frenos del generador \\
\hline & \multirow{3}{*}{$\begin{array}{l}\text { Sincroniz } \\
\text { ación del } \\
\text { generado } \\
\text { r a la red }\end{array}$} & \multirow{2}{*}{$\begin{array}{c}\text { Llevar el } \\
\text { generador a las } \\
\text { condiciones } \\
\text { óptimas de acople } \\
\text { al sistema } \\
\end{array}$} & $\begin{array}{l}\text { Ajustar la velocidad del generador y llevarlo a la } \\
\text { frecuencia del sistema }\end{array}$ \\
\hline & & & Ajustar el voltaje del generador igual al de la red \\
\hline & & $\begin{array}{l}\text { Sincronizar el } \\
\text { generador a la red }\end{array}$ & Orden de cierre al interruptor del generador \\
\hline
\end{tabular}

\subsection{Especificación de operaciones}

Con base en el análisis de la operación del grupo, se condensan las secuencias en detalle, según la metodología para los proyectos de automatización [16].

Las secuencias se organizan por etapas a realizar en un orden específico. Estas etapas representan actividades que resultan en un cambio del material.
Después, se definen las operaciones y sus acciones correspondientes. Estas desarrollan unas acciones para llevar a cabo la operación del proceso.

En la Tabla 1, se muestran las etapas más importantes para los procesos de arranque y sincronización. En Tabla 2 se ilustra las etapas de parada del generador. 


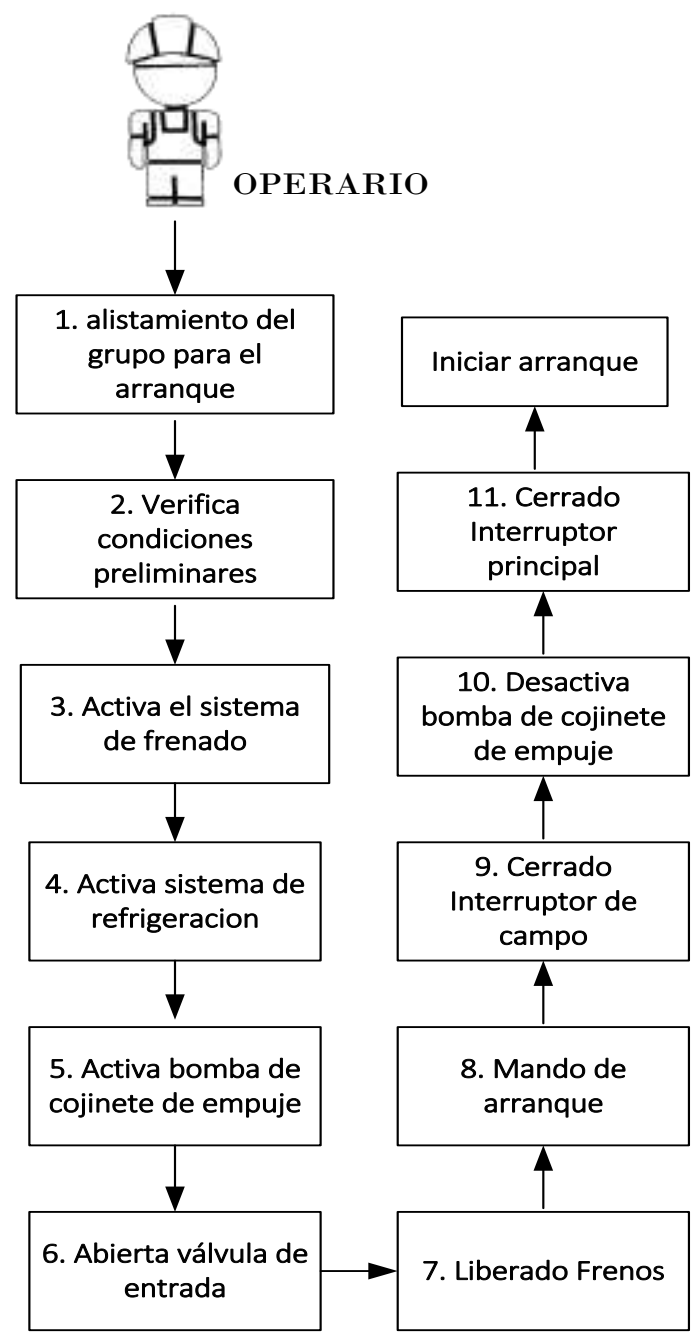

Figura 5. Pasos para el arranque del grupo.

\section{REQUERIMIENTOS HARDWARE Y} SOFTWARE DEL SIMULADOR

En esta parte se utiliza la norma [16] para establecer las características de los requerimientos del simulador. También, se describe la estructura para el simulador de entrenamiento completo y cada una de sus partes, lo mismo que las funcionalidades principales, mediante los diagramas del lenguaje UML (Lenguaje Unificado de Modelado) [17].

Según la norma, los requerimientos se pueden entender como una descripción del sistema a realizar, desde el punto de vista de las necesidades y deseos que se pretende haga el simulador de entrenamiento con respecto al entorno al cual estará asociado. Los requerimientos se dividen en funcionales y no funcionales. Los funcionales son todas las funcionalidades que debe satisfacer el simulador para cumplir con las necesidades de los usuarios mientras que los no funcionales, son todas aquellas características que debe tener el simulador para responder de manera adecuada a todos los requerimientos funcionales.

A continuación, se muestra las principales funciones para el simulador completo; por su parte se evidencia los requerimientos no funcionales. El asterisco (*) en el requerimiento indica que es objeto de alcance en este trabajo para la operación de arranque.

\subsection{Requerimientos funcionales para el simulador de entrenamiento}

\subsubsection{Variables y subsistemas.}

- Permitir la autenticación (usuario y contraseña).

- Permitir cambios en los principales equipos y variables del proceso, para realizar pruebas e inducir fallas.

- Permitir al operador e instructor, la supervisión y control de las principales variables que intervienen en las operaciones de arranque, sincronización y parada del grupo de generación.

- Emular los principales subsistemas del proceso de generación.

- Incluir los mandos e indicadores, para las operaciones de arranque, sincronización y parada, del grupo de generación.

- Presentar por medio de indicadores, el estado de los equipos y variables, que intervienen en las operaciones de arranque, sincronización y parada.

\subsubsection{Operación del Proceso.}

- Permitir al operador entrenarse en las operaciones de arranque, sincronización y parada del grupo de generación.

- Incluir la secuencia de eventos para las operaciones de arranque, sincronización y parada del grupo de generación.

- *Presentar que la secuencia de pasos realizada durante las operaciones, es la correcta.

- *Utilizar estructuras de programación, de tal manera que los eventos, se ejecuten siguiendo una secuencia preestablecida.

- Permitir el reporte del histórico de eventos, realizados durante las operaciones.

\subsubsection{Modelos Matemáticos.}

- Incluir los modelos matemáticos de los principales subsistemas, con sus correspondientes variables y rangos de operación.

- Permitir la conexión de los modelos de los subsistemas, con el software del simulador.

- Permitir la inclusión de nuevos modelos o la modificación de los existentes. 
Tabla 2. Parada del generador

\begin{tabular}{|c|c|c|c|}
\hline PROCESO & ETAPAS & OPERACIONES & ACCIONES \\
\hline \multirow{15}{*}{ PARADA } & \multirow{3}{*}{$\begin{array}{l}\text { Desacople del } \\
\text { generador }\end{array}$} & \multirow{2}{*}{$\begin{array}{l}\text { Llevar el generador a } \\
\text { condiciones óptimas }\end{array}$} & Potencia del generador a cero \\
\hline & & & Voltaje del generador a mínimo \\
\hline & & Abrir interruptor principal & Apertura al interruptor principal \\
\hline & \multirow{12}{*}{ Orden de parada } & \multirow{3}{*}{$\begin{array}{l}\text { Desactivar el sistema de } \\
\text { refrigeración }\end{array}$} & $\begin{array}{l}\text { Válvula principal de entrada cerrada y } \\
\text { salida de agua del generador }\end{array}$ \\
\hline & & & Válvulas de agua de sellos y cojinetes \\
\hline & & & Bomba de agua de refrigeración parada \\
\hline & & \multirow{3}{*}{$\begin{array}{l}\text { Sistema hidráulico en } \\
\text { funcionamiento }\end{array}$} & Bomba del cojinete de empuje encendida \\
\hline & & & $\begin{array}{l}\text { Accionamiento de los sensores de nivel } \\
\text { del refrigerador de velocidad }\end{array}$ \\
\hline & & & $\begin{array}{l}\text { Arranque de la bomba de aceite del } \\
\text { regulador de velocidad para el } \\
\text { movimiento de los alabes }\end{array}$ \\
\hline & & \multirow{3}{*}{ Válvula de entrada cerrada } & Válvula principal cerrada \\
\hline & & & Aplicación del sello de servicio \\
\hline & & & By-pass cerrado \\
\hline & & \multirow{3}{*}{$\begin{array}{l}\text { Sistema neumático en } \\
\text { funcionamiento }\end{array}$} & $\begin{array}{l}\text { Accionamiento de los sensores de } \\
\text { presión del regulador de velocidad }\end{array}$ \\
\hline & & & $\begin{array}{l}\text { Compresores de aire de frenos y } \\
\text { reguladores de velocidad encendidos }\end{array}$ \\
\hline & & & $\begin{array}{l}\begin{array}{l}\text { Accionamiento de los frenos del } \\
\text { generador }\end{array}\end{array}$ \\
\hline
\end{tabular}

\subsubsection{Diseño del simulador.}

- Presentar por medio de indicadores, el estado de los equipos y variables, que intervienen en las operaciones de arranque, sincronización y parada.

- Proveer un ambiente SCADA tanto en apariencia como en funciones.

- Permitir al operador de una central hidroeléctrica, interactuar con una réplica del proceso, sin tener que utilizar el proceso real.

\subsubsection{Interfaces de usuario.}

- Disponer de Interfaces Hombre Máquina (HMI) para distintos niveles de ingreso al simulador, por parte del operador e instructor.
- Disponer de Interfaces para las operaciones de arranque, sincronización y parada.

- Disponer de Interfaces en la computadora del operador y en la computadora del instructor.

- Permitir la comunicación entre la computadora del operador y la del instructor.

5.1.6. Gestión de estudiantes.

- Permitir al instructor evaluar al operario, después de interactuar con el proceso.

- Permitir la conexión con el campus virtual, para el manejo de la gestión de estudiantes. 


\subsection{Requerimientos no funcionales para el simulador de entrenamiento}

- Instalar en el computador del entrenador y del operario, un software de programación que sea un SCADA comercial.

- Instalar en los computadores como mínimo el sistema operativo Windows 7, 32-bit.

- Disponer de computadores que tengan como mínimo: en disco duro 250GB, procesador INTEL DUAL CORE 2 4GB, DDR2, 800MHZ, 2 DIMM VOS ypantalla13.3 WXGA LCD.

- Enviar la información a través de internet al campus virtual de Univalle.

- Permitir futuras ampliaciones y actualizaciones.

\subsection{Casos de uso}

Luego de desarrollar los requerimientos, se construyen los casos de uso. Los casos de uso describen la forma en que interactúan el usuario y las interfaces, detallando todo lo que hace el simulador en respuesta a cada una de las acciones del usuario.

\subsubsection{Definición de actores.}

En esta sección de casos de uso, se especifica el tipo de personas que de alguna manera interactúan en alguno de los procesos del simulador.

\subsubsection{Listado de actores.}

A continuación, se describen los actores que interactúan con el simulador de entrenamiento.

- Administrador del simulador: Persona encargada de manejar las operaciones correspondientes a la habilitación de usuarios e inserción de materiales de apoyo y evaluaciones para el simulador de entrenamiento.

- Instructor: Usuario del simulador que tiene como función más importante la de acompañar el desempeño del operador en la actuación sobre el proceso.

- Operador: El operador puede hacer su entrenamiento para manipular y controlar el proceso de generación de energía con la ayuda de las simulaciones y evaluaciones que valoran su aprendizaje.

\subsubsection{Diagrama de casos de uso.}

El diagrama de casos de uso "explica gráficamente el conjunto de casos de uso del simulador de entrenamiento, los actores y la relación entre estos y los casos de uso [17]. En la Figura 6 se muestran los casos de uso para el simulador completo, con base en los requerimientos.

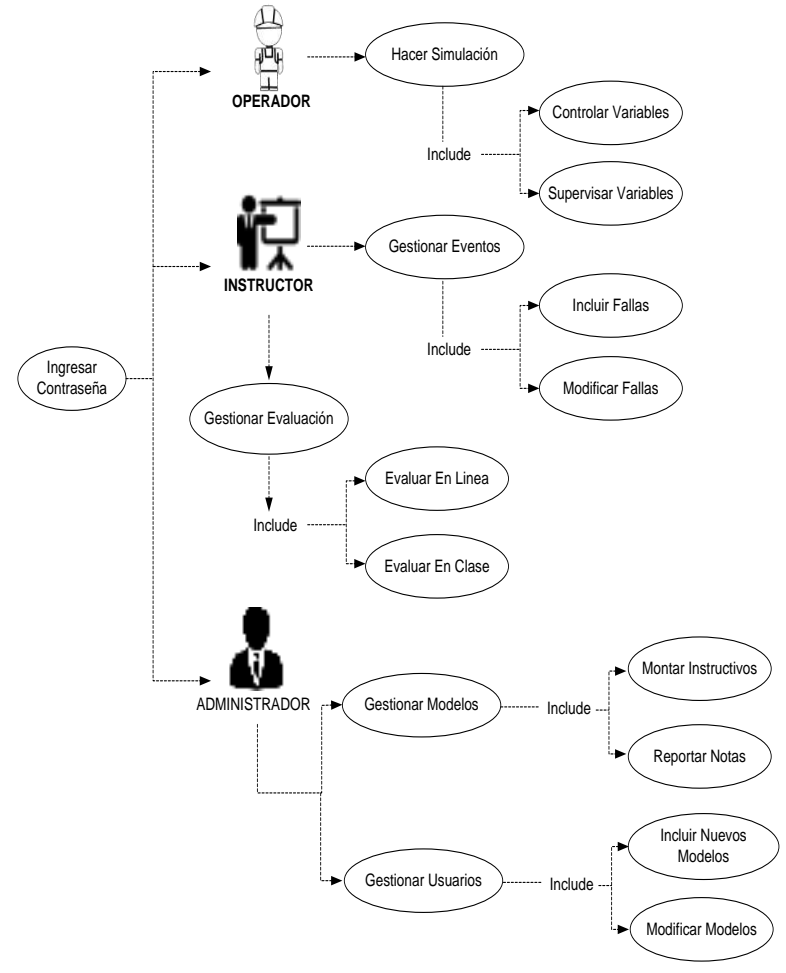

Figura 6. Casos de uso para el simulador completo.

\section{CONCEPCIÓN DEL SIMULADOR DE ENTRENAMIENTO}

En esta sección se presentan las arquitecturas hardware y software para el simulador y las interfaces de interacción con el usuario. Finalmente, se presentan las interfaces realizadas para el arranque de un grupo.

\subsection{Arquitectura hardware y software para el simulador de entrenamiento}

El simulador de entrenamiento de operadores para centrales hidroeléctricas, son todos los componentes hardware y software que tienen como finalidad, permitir supervisar y controlar el proceso de generación de energía. A continuación, se muestran los diagramas que presentan los componentes hardware y software para el simulador.

\subsubsection{Arquitectura Hardware.}

Este diagrama está enfocado específicamente al hardware para el simulador. En la Figura 7 se muestran los componentes del hardware: un computador con aplicaciones que le permiten a un operario ejercer contacto directo con el proceso a través de maniobras, para así realizar el entrenamiento. Además, en otro computador, un instructor con las mismas aplicaciones, realiza la supervisión del desempeño. También, en otro computador el administrador hace la gestión para el 
simulador las comunicaciones de los computadores se realizan por medio del protocolo TCP/IP. Adicionalmente, la gestión de estudiantes se realiza desde un campus virtual.

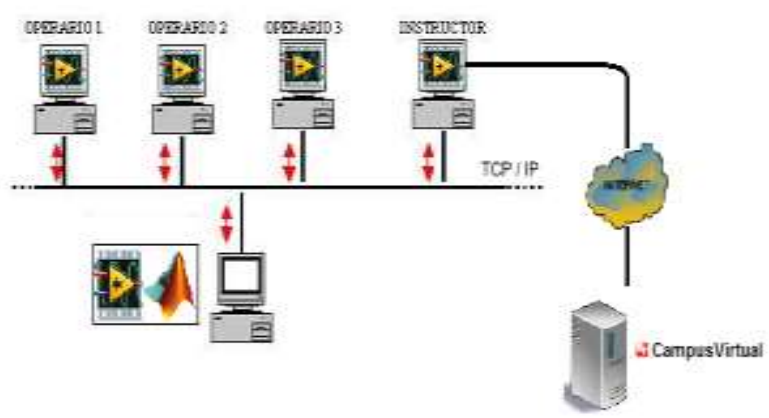

Figura 7. Estructura hardware para el simulador.

\subsubsection{Arquitectura Software.}

En la Figura 8 se muestran los elementos software que se proponen para el simulador. Se escoge como base para el simulador, el software conocido como LabVIEW [18], por tener el comportamiento de un SCADA comercial, por su facilidad de programación, su entorno gráfico para las interfaces Hombre Máquina (HMI) y su compatibilidad de enlace con el programa Matlab; mediante una herramienta de LabVIEW conocida como Simulation Interface Toolkit.

El Matlab [14] es un programa de análisis y simulación de sistemas ampliamente utilizado en la academia con una base de modelos en el toolbox de sistemas de potencia y que se denominan Synchronous Machine pu Standard.

En trabajo previo [9] realizado en la universidad del valle se acondicionaron los modelos del sistema de control de velocidad y excitación mediante el programa Matlab y como LabVIEW no cuenta con un toolbox de sistemas de potencia, se usaron en este trabajo. Esto, para que las interfaces del simulador de entrenamiento desarrolladas en este trabajo, tengan un comportamiento en tiempo real y similar al proceso de generación de energía.

\subsection{Interfaces Hombre Máquina (HMI)}

Como el simulador de entrenamiento debe emular el proceso de generación de energía eléctrica de una central hidroeléctrica, esta emulación debe ser un sistema SCADA. Para estructurar un SCADA, se tiene en cuenta pantallas de Interfaces Hombre Máquina (HMI) para distintos niveles de ingreso, que presentan información de los principales sistemas y variables, por medio de imágenes y texto.

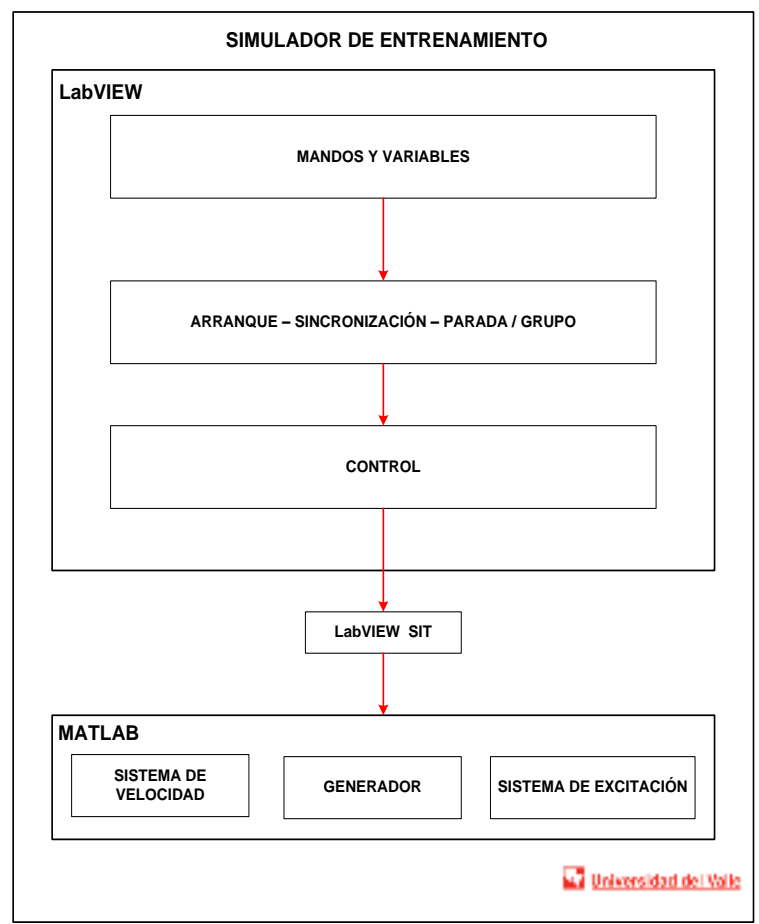

Figura 8. Estructura Software para el simulador.

En la Figura 9 se presenta la arquitectura para los niveles que se proponen en este trabajo de investigación del simulador completo y para el arranque del grupo, que se hace en este trabajo.

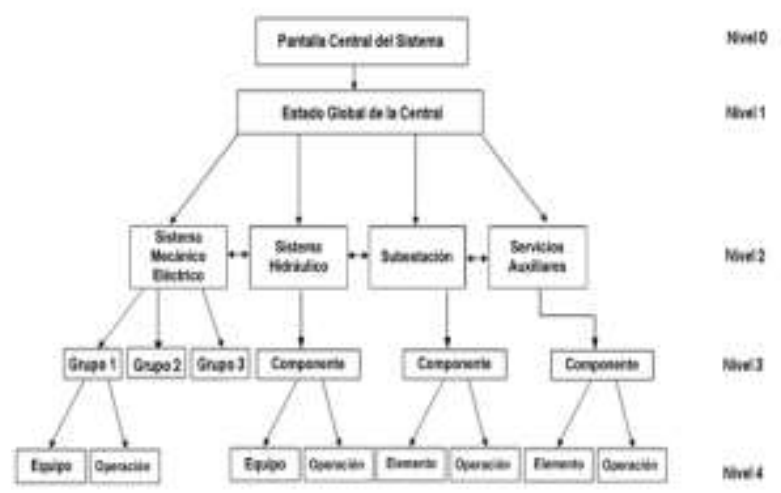

Figura 9. Niveles para el SCADA.

\subsubsection{Pantallas SCADA.}

\section{- Pantalla tipo nivel 0}

Se expone una pantalla con la imagen de la central y en la parte inferior se cuenta con iconos de acceso al simulador y a diferentes pantallas del proceso de 
generación de energía. En el documento escrito, se presenta el ejemplo de cómo debe ser esta pantalla.

\section{- Pantalla tipo nivel 1}

En ella se pueden apreciar las variables principales que se deben supervisar y monitorear continuamente como: nivel del embalse, abertura de compuertas, caudal vertido, caudal total, potencia entregada, entre otros). En el documento escrito, se presenta el ejemplo de cómo debe ser esta pantalla.

\section{- Pantalla tipo nivel 2}

Para este nivel, se deben realizar pantallas para los sistemas que se ilustran en la Tabla 3. En el documento escrito, se presentan ejemplos de cómo deben ser las pantallas para estos sistemas.

\section{- Pantalla tipo nivel 3}

Se realiza en este trabajo para la supervisión y monitoreo de las variables de un componente (grupo1, grupo 2, grupo 3...), con base en trabajos realizados [19]. Se hace en este trabajo y se ilustra en la Figura 10.

Tabla 3. Componentes para el nivel 2.

\begin{tabular}{|c|c|}
\hline SUBSISTEMA & COMPONENTE \\
\hline \multirow{6}{*}{ Mecánico Eléctrico } & Generador \\
\hline & Refrigeración \\
\hline & Turbina \\
\hline & Sistema de Regulación \\
\hline & Frenos \\
\hline & Válvula de Entrada \\
\hline \multirow{4}{*}{ Subestación } & Transformador \\
\hline & Líneas \\
\hline & Barraje \\
\hline & Transformadores SS.AA \\
\hline \multirow{2}{*}{ Servicios } & Refrigeración \\
\hline & Alimentación AC \\
\hline Auxiliares & Alimentación DC \\
\hline \multirow{3}{*}{ Hidráulico } & Embalse \\
\hline & Sistemas de protección \\
\hline & Descarga \\
\hline
\end{tabular}

\section{- Pantalla tipo nivel 4.}

Se realiza en este trabajo para la operación de un grupo según [19] y se muestra en la Figura 11. La pantalla para el arranque también se hace en este trabajo y se ilustra en la Figura 12.

\subsection{Caso de implementación}

A través del caso de implementación, se evalúa el comportamiento de las interfaces del simulador de entrenamiento desarrolladas en este trabajo para el arranque de un grupo de generación, al ser enlazadas mediante una herramienta de LabVIEW conocida como Simulation Interface Toolkit con los modelos del sistema de control de excitación y con los modelos del sistema de velocidad. Los modelos de simulink generan un código LabVIEW que asocia controles e indicadores con las pantallas de LabVIEW. A continuación, se presenta la prueba realizada a las interfaces.

\subsubsection{Prueba de funcionamiento-arranque de un grupo de generación.}

\section{Requerimientos:}

1. Disponer de la Interfaz para la operación de arranque.

2. Incluir los mandos para la operación de arranque.

3. Presentar por medio de indicadores, el estado de los equipos y variables.

4. Permitir al operador entrenarse en la operación de arranque.

5. Presentar que la secuencia de pasos durante la operación de arranque, es la correcta.

6. Utilizar estructuras de programación, de tal manera que los pasos, se ejecuten siguiendo una secuencia preestablecida.

7. Incluir los modelos matemáticos de los principales sistemas que intervienen en el arranque, con sus correspondientes variables y rangos de operación.

8. Permitir la conexión de los modelos de los principales sistemas que intervienen en el arranque, con el software del simulador.

Hardware requerido: Computador

Software Requerido: LabVIEW-Matlab

Objetivo: Probar el correcto funcionamiento de las pantallas en el cumplimiento de la secuencia de pasos para realizar el arranque del grupo.

Datos de la prueba. Se utilizará la secuencia descrita en 4.1 . 


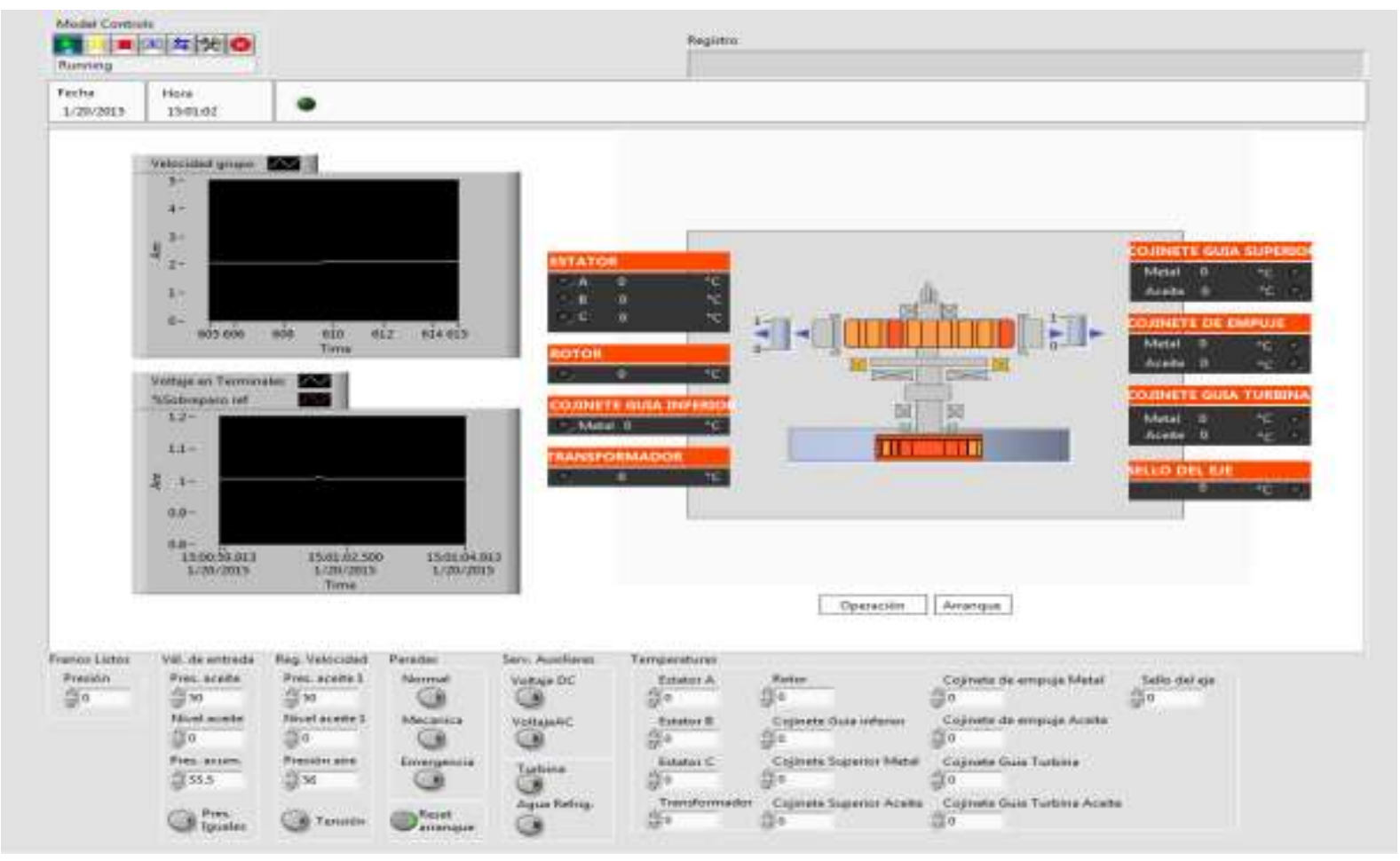

Figura 10. Pantalla tipo nivel 3.

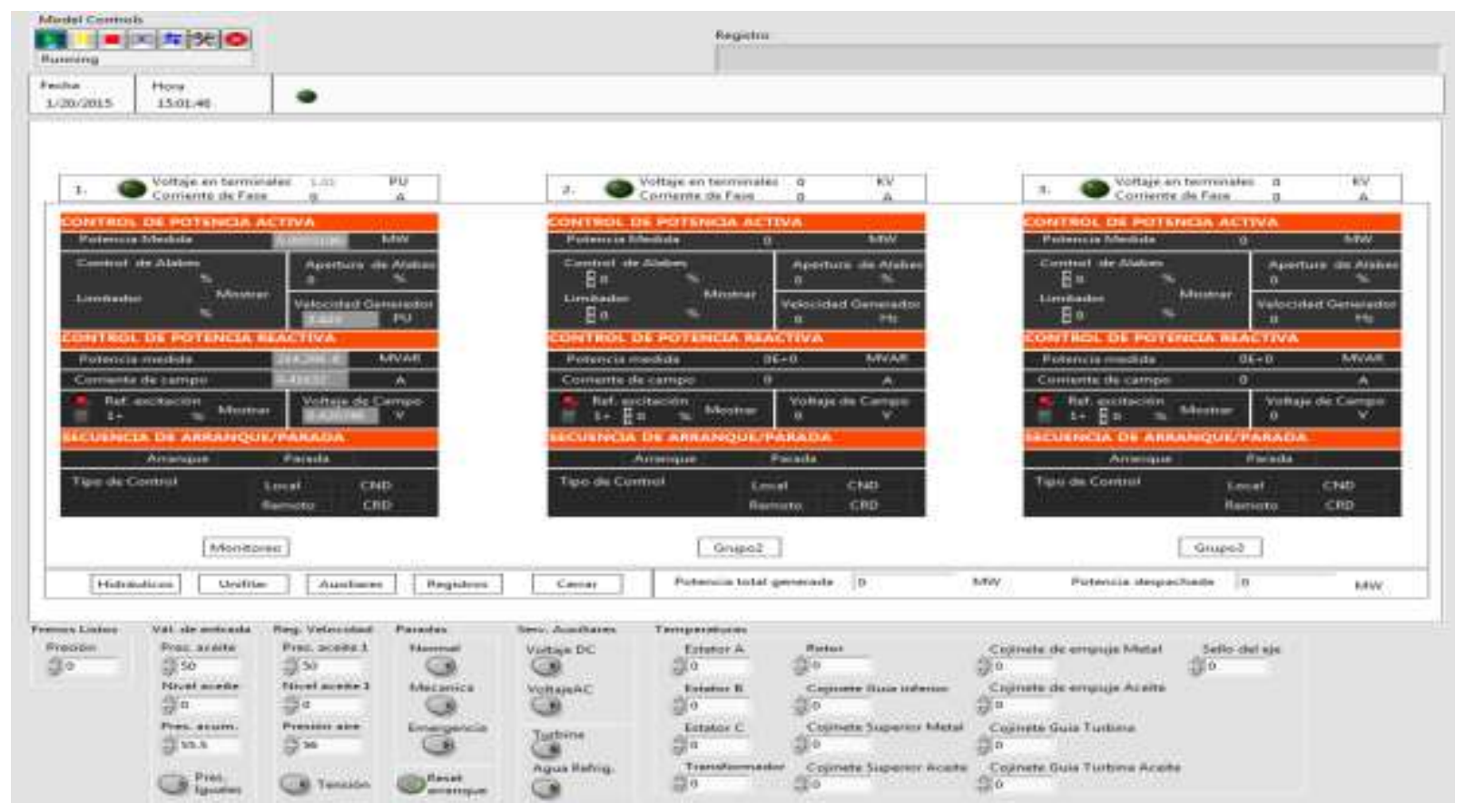

Figura 11. Pantalla tipo nivel 4. 


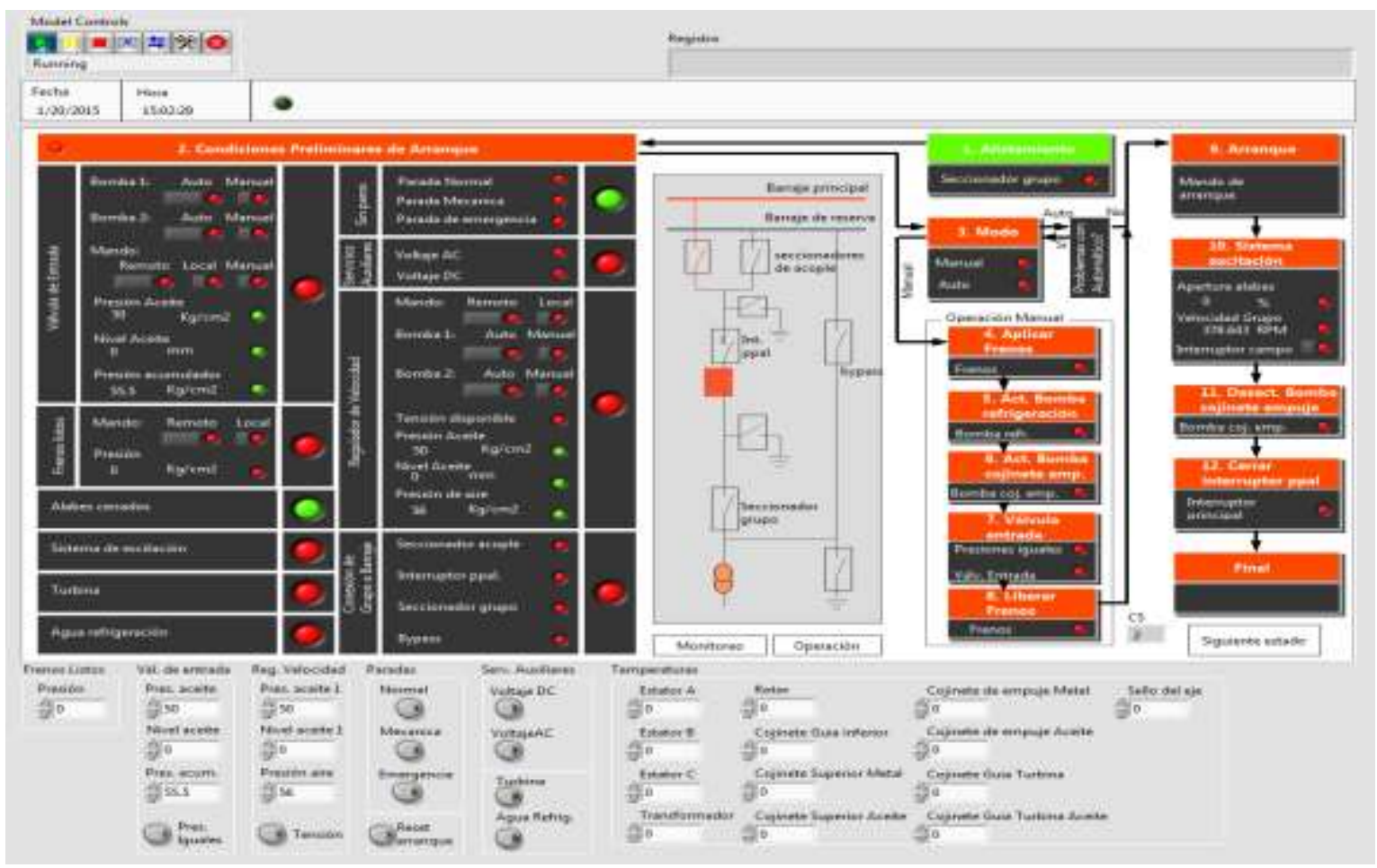

Figura 12. Pantalla para el arranque.

\section{Procedimiento:}

1. Se abre la pantalla de arranque y se apertura el seccionador del grupo.

2. Se continúa con la revisión de las condiciones preliminares.

3. Después del cumplimiento de las condiciones preliminares, de forma visual se muestra la secuencia a cumplir para el arranque manual.

4. Es importante para el cumplimiento de la secuencia de arranque, chequear el estado de los equipos y rangos de las variables.

\subsubsection{Resultados obtenidos}

- Se puede observar la Interface para el arranque del grupo. En esta, se puede apreciar los mandos para el arranque. También, indicadores que ilustran el estado de los equipos y variables.

- La pantalla de arranque muestra la secuencia de pasos para realizar este. Es posible observar que se utilizan estructuras de programación, de tal manera que los pasos, se ejecuten siguiendo una secuencia preestablecida.

- Las pantallas se conectan con los modelos matemáticos de los principales subsistemas lo mismo que con modelos de primer orden para los subsistemas que no cuentan con modelado matemático.

\section{CONCLUSIONES Y OBSERVACIONES}

- En la concepción del simulador, se han integrado las metodologías del desarrollo de proyectos de automatización y de software. Adicionalmente, se hace uso de los principales modelos para el sistema de generación de energía eléctrica.

- El diseño realizado para cumplir los requerimientos, utiliza el SCADA Labview para las HMI y el Matlab para la ejecución de los modelos. Se propone la estructura general de las pantallas SCADA a implementar en el simulador.

- La concepción general se desarrolla y prueba para el caso del arranque de un grupo de generación; se plantea por lo tanto la metodología y estructuración general para desarrollar el simulador. 


\section{AGRADECIMIENTOS}

Al Doctor José Miguel Ramírez Scarpetta, por bridarme parte de su tiempo y sabiduría en los momentos en los que más necesitaba de una persona que orientara mí trabajo de investigación. A la Institución Universitaria Antonio José Camacho por el tiempo brindado para avanzar en el trabajo de grado. A los ingenieros Fabián González, Javier Valencia, Adolfo Hoyos, William Díaz, Andrés Julián Saavedra, Cesar Romero, Diego López. También, Katherine Agudelo, Marlon Aldana y a todos aquellos que de alguna forma apoyaron este trabajo.

\section{BIBLIOGRAFÍA}

[1] V. Cutsem, "A new training simulator for improved voltage control of the hydro-québec system," IEEE, Canada, 2004.

[2] Vásquez, Ana et al., "Simuladores de unidades de generación eléctrica via web,” México, 2015.

[3] T. e. Gomez, "Simulador de entrenamiento y certificación, para operadores de cento de control," Argentina, 2005.

[4] G. Romero, "Development of a 25MW geothermal power plant full scope simulator," IEEE, Mexico, 2009.

[5] Indra, "Simulador de entrenamiento del sistema de potencia colombiano," Medellin, 2012.

[6] Zhuravlevy y Andreev, "Development of a full scale training simulator for an $800 \mathrm{MW}$ power unit," Rusia, 2013.

[7] L. Xianshan, "Full scope real-time simulation of hydropower plant for a training and research simulator," 2005.

[8] J. Garrido y et, "Modelado y simulacion de centrales hidráulicas," Argentina, 2007.

[9] M. Fabio, "Sistema de entrenamiento de operadores de plantas de genración eléctrica," Universidad del Valle, Santiago de Cali, 2011.

[10] J. A. Saavedra, Modelado para estudios de estabilidad de los sistemas de control de velocidad y excitación de la central de salvajina, Cali: Universidad del Valle, 2002.

[11] F. Bravo, "Funcionamiento de equipos y sistemas de centrales del cauca," La central, Cauca, 2008.
[12] A. Saavedra, "Validación de los modelos de los sistemas de control de generación para estudios de estabilidad de la central de Salvajina; parte I: control de velocidad," Energia y computación, vol. 11, n 2, pp. 60-65, 2004.

[13] A. Saavedra, "Validación de los modelos de los sistemas de control de generación para estudios de estabilidad de la central hidroeléctrica de Salvajina. Parte II. Control de excitación.," Energía y Computación, vol. 11, nº 2, pp. 66-71, 2003.

[14] Matlab, "Software de simulación Matlab. The lenguage of technical computing. Versión 7.13," 2011.

[15] EPSA, "Instructivo de operacíon de un grupo de generación," EPSA, Cali, 2008.

[16] J. M. Ramírez S., "Sistemas de control-Enfoque de Proyectos," Universidad del Valle, Cali, 2014.

[17] IEEE, «Recomended practice for software requirements specifications 830,» IEEE, E.U.A, 1998.

[18] J. Schmuller, Aprendiendo UML, Mexico: Prentice Hall, 2013.

[19] LabVIEW (Laboratory Virtual Instrumentation Engineering Workbench), 2012.

[20] P. Thomasset, "Instalación de sistema SCADA C.H.Dr. Terra y Rio Negro," IEEE, Uruguay, 2010.

[21] I. P. E. Society, "Standard definitions for excitation systems for synchronous machines," U.S.A., 2007.

[22] S. Mayolo, J. Tavira, A. Vásquez y J. Vergara, "Simuladores de unidades de generación eléctrica accesibles vía web," Boletin IIE, pp. 144-147, Julio 2015.

[23] E. Burgos y I. Rios, "Simulador de rodado de turbina para el adiestramiento de operadores," Boletin IIE, pp. 167-171, Julio 1993. 